\title{
Development of automatic intercomparison system for generation of time scale ensembling several atomic clocks
}

\author{
P.P. Thorat ${ }^{1 \star}$, A. Chatterjee ${ }^{1}$, P. Kandpal ${ }^{1}$, M.P. Olaniya ${ }^{1}$, A.K. Suri ${ }^{1}$, A. Sen Gupta ${ }^{1}$, and P. Banerjee ${ }^{2}$ \\ 1 National Physical Laboratory (CSIR-NPL), 110012 New Delhi, India \\ 2 ECE Dept, ASET, Amity University UP, 201303 Noida, India
}

Received: 24 September 2014 / Accepted: 15 January 2015

\begin{abstract}
National physical laboratory India (NPLI) has five commercial cesium atomic clocks. Till recently one of these clocks had been used to maintain coordinated universal time (UTC) of NPLI. To utilize all these clocks in an ensemble manner to generate a smoother time scale, it has been essential to inter-compare them very precisely. This has been achieved with an automatic measurement system with well-conceived software. Though few laboratories have developed such automatic measurement system by themselves based on the respective requirements; but they are not reported. So keeping in mind of the specific requirement of the time scale generation, a new system has been developed by NPLI. The design has taken into account of the associated infrastructure that exists and would be used. The performance of the new system has also been studied. It has been found to be quite satisfactory to serve the purpose. The system is being utilized for the generation of time scale of NPLI.
\end{abstract}

Keywords: Coordinated universal time (UTC), time scale, intercomparison, atomic clocks, H-maser

\section{Introduction}

Commercially available cesium atomic clocks are very close realization of definition of the second in the international system of units (SI). So, most of the time keeping laboratories of the globe maintains the national time with the help of commercially available cesium atomic clock. Many timing laboratories have more than one commercial cesium clocks, few might have Hydrogen maser also. Such clocks are individually fairly stable clocks. But physical devices can fail, so many laboratories which can afford, prefer to keep several clocks. These are together treated as an ensemble. Intercomparison of the clocks may be combined through suitable algorithm to raise the performance of the ensemble than that by any individual clock. National physical laboratory India (NPLI) has five commercial cesium atomic clocks and one H-maser. One of these clocks maintains coordinated universal time (UTC) [1] of NPLI. It is being planned to utilize all these clocks in an ensemble manner to generate a smoother time scale. For optimum utilization of these clocks it is essential to inter- compare them very precisely. However, these intercomparisons have to be done at a regular interval of time without interruptions irrespective of the holidays and office hours of laboratories. This has been achieved with an automatic measurement system with well-conceived software. The configuration of this arrangements require a system which automatically selects a pair of clocks in se-

* Correspondence: pranuthorat@gmail.com quence, a precise time interval counter which compare a selected pair of clocks, and a computer which records these data and store them. Few laboratories have generated respective time scales [2-4]. All of them have developed such automatic measurement system by themselves based on the respective requirements. No report on the development of such system is available.

In view of the above, such system has been developed by NPLI and its performance has also been studied. This paper elaborates the design scheme, presents the results of the evaluation of its performance and emphasizes its salient features.

\section{Design concept of switching system}

In order to implement the arrangement for the execution of automatic intercomparison of a pair of atomic clocks a set-up as shown in Figure 1 is designed. The important components of this scheme are a precise time interval counter (TIC) and the switching system for the automatic selection of pair of clocks. Precise TIC of 50 ps resolution is used for this measurement. Switching system as required for this purpose is not available and has to be developed by NPLI. Based on the requirement shown in Figure 1 the design of the switching system has been planned considering the following aspects.

- The time delay of the clock pulses through the switching system should be as minimum as possible. 


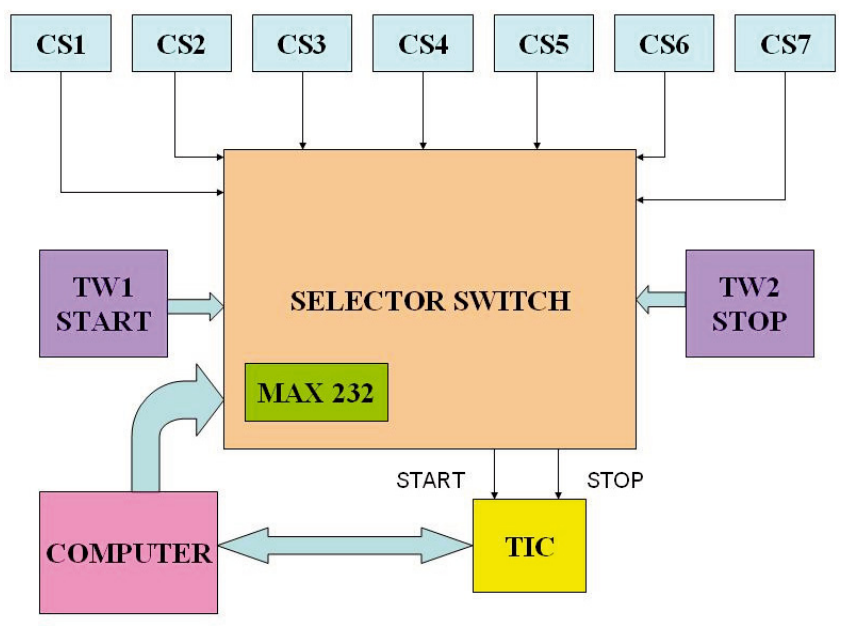

Fig. 1. Scheme for automatic measurement system.

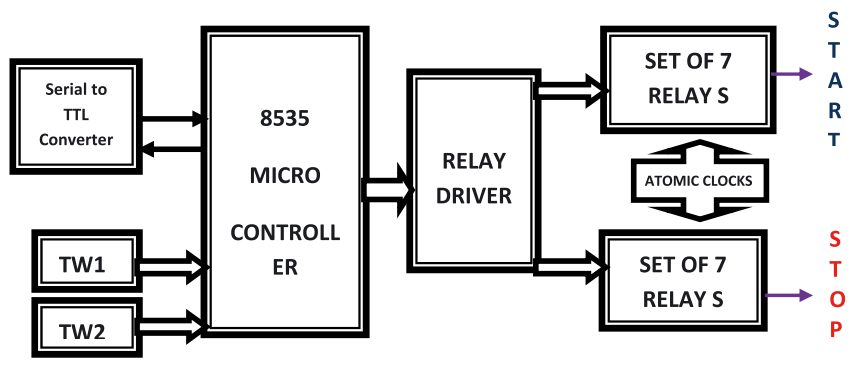

Fig. 2. Functional block diagram of switching system.

- The time delay involved should be constant i.e. it should not change with time. This pre-requisite eliminates the use of any active components, solid state relays or logic gates in the system. This is because the delay involved with them is not uniform and not stable. But, the solid state relay operates very fast. The particular application does not require the fast operation. So mechanical reed relays are used in the system. They do not change with time.

- A provision should be available to indicate which of two clocks have been selected for measurement and given to start and stop ports of TIC, respectively.

- The system should be programmable so that the entire process of taking the measurement may be done according to the pre-decided schedule automatically. However, it should also have the flexibility to operate the system manually so that readings can be taken even if the system is not being controlled by a computer.

\section{Development of switching system}

Keeping in mind the considerations mentioned in Section 2 the scheme of design of switching system has been configured as shown in Figure 2. One pulse per second (1 pps) from all seven cesium atomic clocks are fed into the selector switch whose function is to select one pair of clocks and connect them to TIC.
Selector switch selects any two of the seven clock pulses by activating the corresponding relays which connects/disconnects the respective mechanical switch.

Output ports of the selector switch remains connected to start and stop inputs of TIC respectively. It can either be done manually by using two thumbwheel switches for start and stop or automatically using a software program.

The outputs of selector switch are fed into the time interval counter whose function is to measure the delay between start and stop pulses. The data from the time interval counter can also be stored in the computer through a specially developed program.

The system is based on 8535 microcontroller. It basically drives one pair of relay drivers based on external command. This external command may be fed by thumbwheel switches or through a computer interfaced RS232 port of switching system. The configuration of switches of the respective relays is shown in Figure 3. Each relay has two switches; one of them gives path for cesium clock input to START or STOP output whereas the other one drives the respective LED to give visual indication on the status of the connectivity.

The microcontroller is used to select the desired pair of atomic clocks. One port of eight pins of the microcontroller is connected to the thumb wheel pair TW1 and TW2. The outputs from the microcontroller are fed to corresponding 14 relay drivers to activate selected two relays out of 14 relays.

Software through PC selects one particular pair of relay drivers at a pre-programmed time. The relay drivers produce a low output current which ultimately drives the relays to select the desired pair of atomic clocks. Serial "COM" port of $\mathrm{PC}$ is connected to microcontroller through ICMax232 - RS232C/TTL converter interface to send command to Switching system.

The front view and rear view of the completely developed system are hown in Figures $4 \mathrm{a}$ and $4 \mathrm{~b}$, respectively. Figure $4 \mathrm{c}$ shows the component layout of the unit.

\section{Evaluation of performance of the system}

The important aspects of the performance of the switching system are the following:

1. To determine the delay of each of the paths from the inputs to the start and stop outputs.

2. To evaluate the constancy or variability of these delays.

The above performance has been evaluated by the following precise arrangement. The input that will be fed to the one of the seven input ports would travel to the start or the stop port during the utilization of the system. So we are supposed to measure fourteen (14) path delays between start/stop port and input channel ports.

This very precise determination of path delay is not a straightforward method. It is difficult to isolate the delay of the cables and connectors while they are used in the process of measurement of the path delays. So a new scheme has been worked out to determine the channel delay in two 
P.P. Thorat et al.: Development of automatic intercomparison system for generation of time scale ensembling... 106-p3

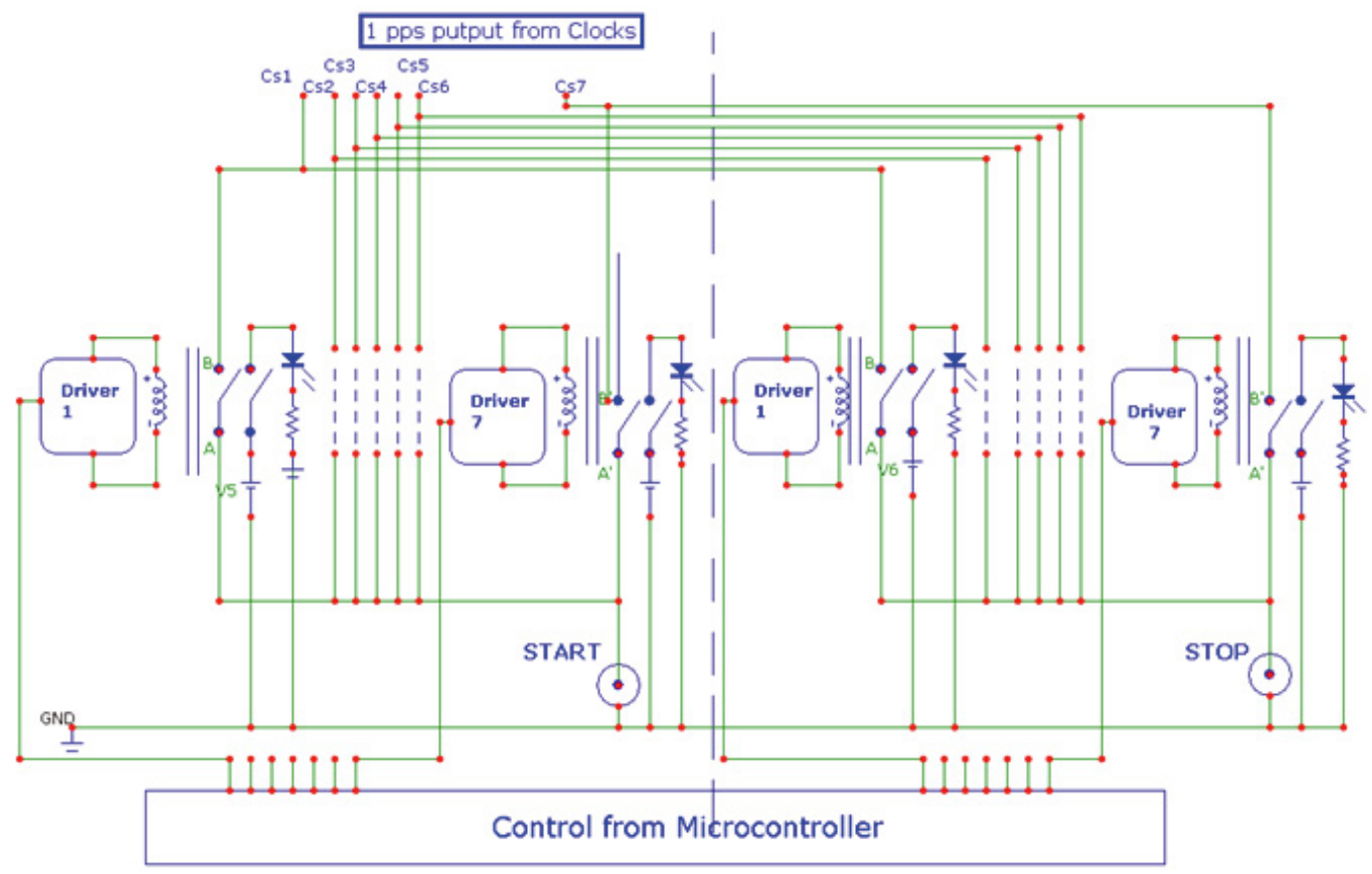

Fig. 3. Connection configuration of relay switch.

phases of measurements. This scheme measures the delays in two phases as explained in the following subsections.

\subsection{Measurement 1}

Let us assume that a Cesium clock is connected to any of the input port using $\mathrm{T}$ connector (Figs. $5 \mathrm{a}$ and $5 \mathrm{~b}$ ). The $\mathrm{T}$ connector may be represented by three arms (ao, ob and oc) as shown in Figure 5b.

One of three ends of $\mathrm{T}$ connector is connected to the Cesium clock via cable and one to the input of the switching system and the third end of $\mathrm{T}$ connector is connected to START port of TIC via cable. The output of selector switch (Start or Stop port) is given to STOP terminal of TIC.

Hence the delay that TIC records is

$$
\text { Meas } 1=(y+z+x 2)-(x+x 1),
$$

where

$x=$ delay of the cable \#1 used at the output end of SS,

$z=$ internal path delay of SS (to be determined),

$y=$ delay of the cable \#2 used at the input of SS,

$x 1=$ delay in the length "ao" of T connector as shown in Figure 5b,

$x 2=$ delay in the length "oc" of T connector as shown in Figure 5b.

\subsection{Measurement 2}

Let us consider other case in which the arrangement is exactly similar to Figure 5a except that cables "\#1" and "\#2" have been swapped as shown in Figure 6 .
The measurement in TIC is referred as measurement 2 and may be given as,

$$
\text { Meas } 2=(x+z+x 2)-(y+x 1) \text {. }
$$

Now if we combine equations (1) and (2), we get,

$$
\begin{aligned}
\text { Meas } 1+\text { Meas } 2= & y+z+x 2-x-x 1+x \\
& +z+x 2-c-x 1 \\
= & 2 z+2 x 2-2 x 1=2\{z+(x 2-x 1)\} .
\end{aligned}
$$

Or

$$
(\text { Meas } 1+\text { Meas } 2) / 2=z+(x 2-x 1) .
$$

Considering the basic design of normal T-connector, it may be quite logically assumed that the delays of paths "oa" and "oc" are almost equal. Thus, $(x 2-x 1)$ becomes zero and RHS of equation (3) reduces to ' $z$ ' only, giving the respective required path delay.

By this method precise path delays which are to be used in the generation of time scale could be measured.

\section{Utilization of the system}

The complete automatic measurement system based on the newly developed selector unit has been in operation in NPLI and is shown in Figure 7. Based on this system NPLI has already started taking regular measurement to inter compare clocks. To make the system automatic the necessary software has been developed. The software is user friendly.

It has the provision to pre-program the selection of time interval after which each phase of measurement 


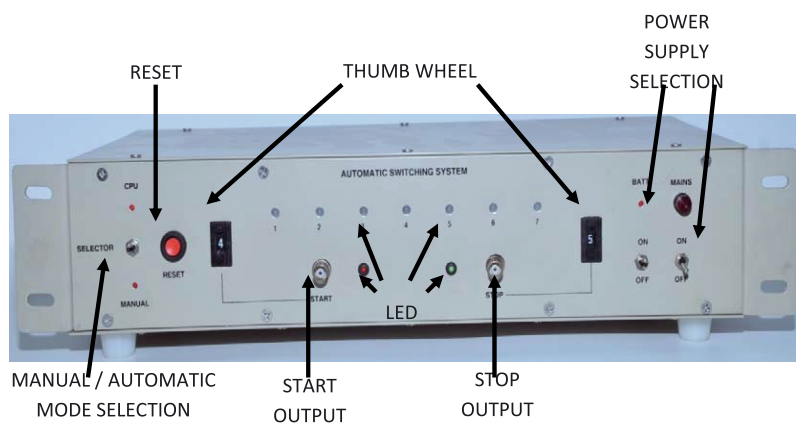

(a)

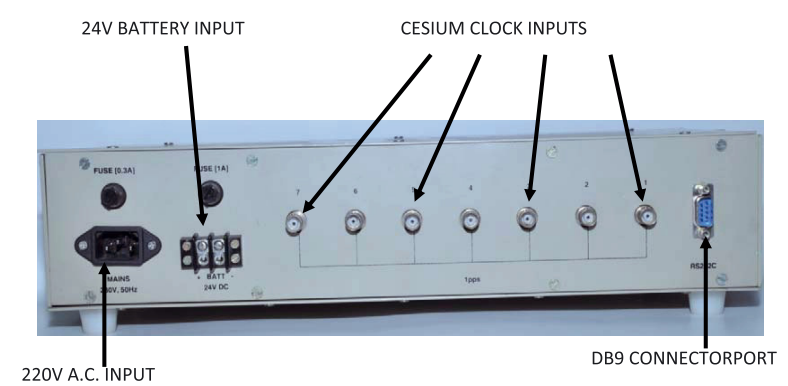

(b)

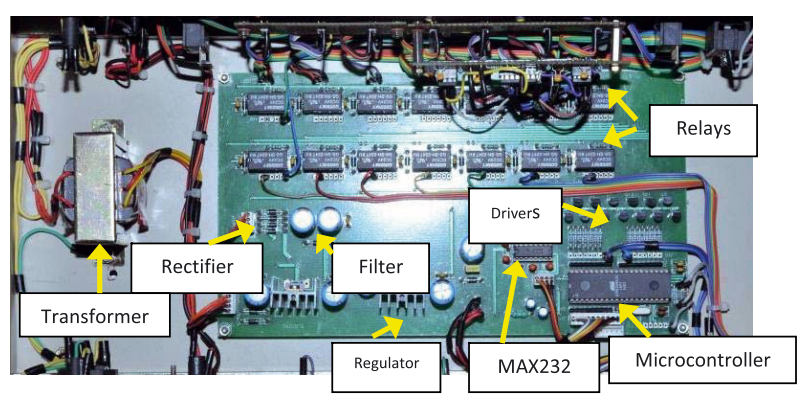

(c)

Fig. 4. (a) Front view of the switching system. (b) Rear view of the switching system. (c) Component Layout of switching system.

has to be repeated, to select the clock pairs for intercomparison and also to identify the sequence of pairs for measurement. After execution of program the measurement continues. It selects the pair of inputs; the data of TIC is fetched into the computer for storing in computer with time tag and pair identifier tag. The flow chart of the software [4] is shown in Figure 8. The desired pair is selected at least one second before the scheduled measurement. This eliminates the chance of any effect of mechanical jitter during the process of relay connection which lasts only for few milliseconds. Each set of measurement is taken at least twenty times to reduce the effect of triggering noise.

The two sets of measurement are to be carried out for each of 14 paths (e.g. channel 1 port to START, channel 1 port to STOP port, channel 7 port to START port, channel 7 port to STOP port). These measurements were carried out for each path. Each set of measurements have been taken at least 20 times. Out of each set of mea-

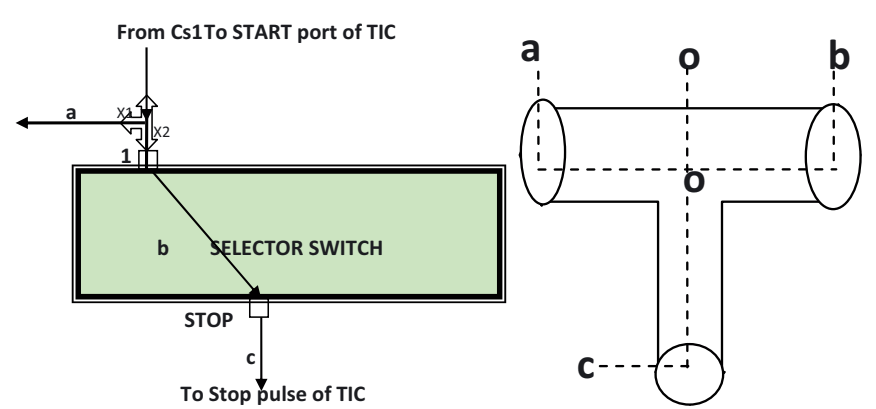

(a)

(b)

Fig. 5. (a) Forward path measurement. (b) T connector.

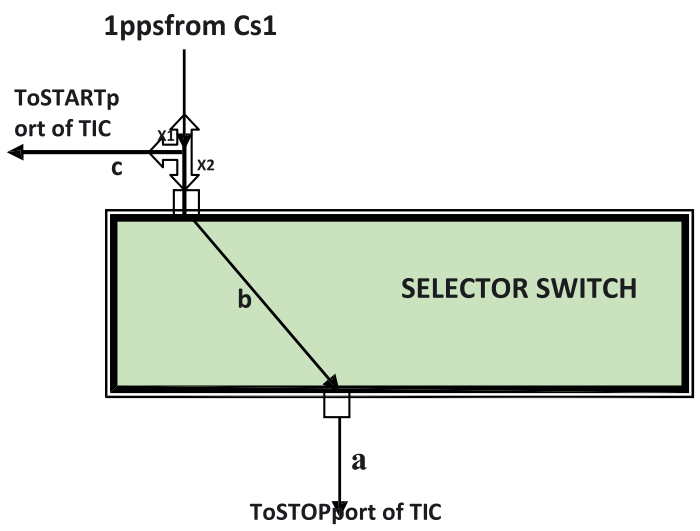

Fig. 6. Measurement 2.

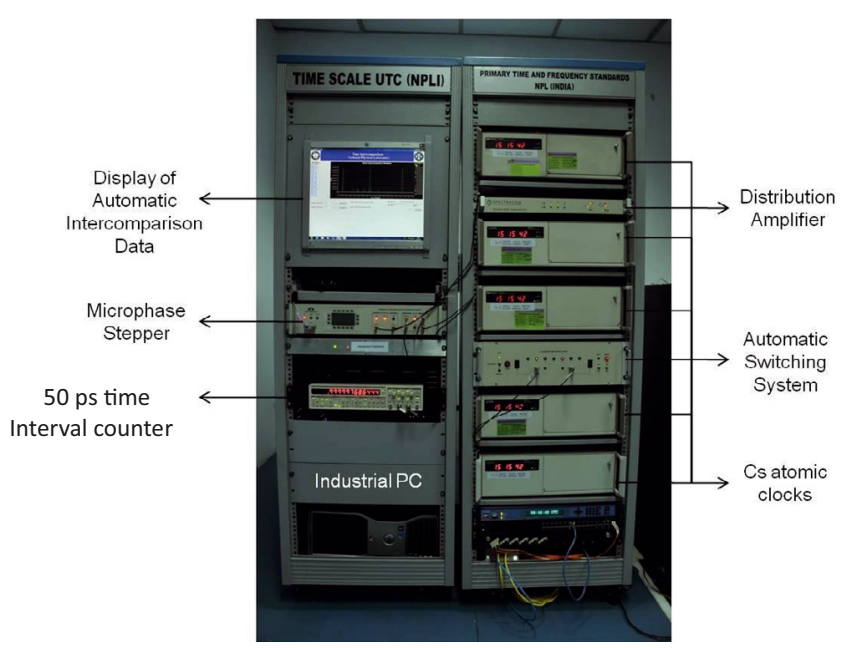

Fig. 7. Automated data acquisition system for time scale generation.

surement, mean value and corresponding standard deviation (SD) are found out. These measurements are taken regularly in time scale generation system which is being operated in a normal environmental condition. Mean value of each set indicates the absolute value of the delay and the corresponding SD shows the uncertainly of the measurement.

General observations confirm that absolute value of the delay for the respective channel lie within a range 
P.P. Thorat et al.: Development of automatic intercomparison system for generation of time scale ensembling... 106-p5

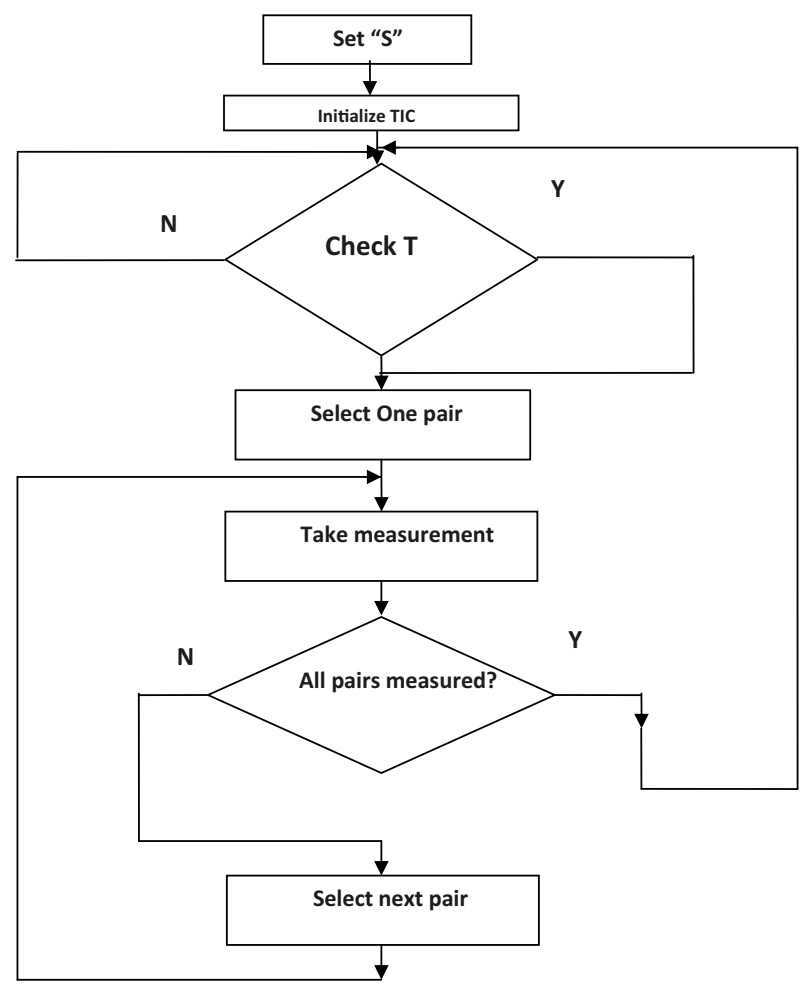

Fig. 8. Flow chart of the software. $\mathrm{S}=$ No of data for each measurement (e.g. 1 or 10) for averaging. S should be a multiple of $10 . T=0 \mathrm{~h}$ or $4 \mathrm{~h}$ or $8 \mathrm{~h}$ or $12 \mathrm{~h}$ or $16 \mathrm{~h}$ or $20 \mathrm{~h}$. TIC initialize $=1$. Mode set in time interval mode. 2 Port $\mathrm{A}$ in Start. Port B in Stop.

from $7 \mathrm{~ns}$ to $8 \mathrm{~ns}$. It is interesting to note that the variability (i.e. standard deviation SD) of the delay of each channel is quite insignificant and they are limited to less than 100 ps. These values of the channel delay have been used for the generation of time scale of NPLI.

\section{Conclusion}

Design and development of selector switching system have been completed. Its performance has been found to be quite satisfactory and it adequately serves the purpose for generation of time scale. The standard deviation of the channel delays have been found to remain well below 100 ps over a large set of observations. This could be achieved because no active circuits or logic gates were used in the designed system. The system is in regular use and found to be quite reliable. Recently the time scale as reported in BIPM circular $\mathrm{T}$ has shown significant improvement in uncertainty values. This is the result of few measures taken by NPLI including the commissioning of this automatic measurement system.

\section{References}

1. P. Tavella, T. Claudine, Comparative Study of Time Scale Algorithm, Metrologia 28, 57-63 (1991)

2. Y. Hanado, M. Imae, M. Aida, M. Hosokawa, H. Ito, F. Nakagawa, Y. Shimizu, Algorithm of ensemble of atomic time, J. NICT 50, 157-167 (2003)

3. Y. Hanado, M. Hosokawa, K. Imamura, N. Kotake, Improvement of frequency changes at Japan standard Time, Electr. Eng. Jpn 157, 29-37 (2006)

4. P.P. Thorat, P. Banerjee, Software in $\mathrm{C}++$ for Automatic recording of data for intercomparison of pair of clocks through time interval counter after sequentially selecting pairs of clocks through selector unit (Copyright applied) 